\title{
EVOLUÇÕES DE ECUS FATEC USP
}

\author{
Valter Manuel Jardim da Mata, Armando Antônio Maria Laganá, Bruno Silva Pereira, \\ Demerson Moscardini
}

Escola Politécnica da Universidade de São Paulo

E-mails: valtermanuel@gmail.com, lagana@1si.usp.br, bruno.sp@usp.br, demersondonoc@hotmail.com

\section{RESUMO}

Atualmente, os motores a combustão interna são amplamente utilizados em carros de passeio, motocicletas, veículos de transporte e motores estacionários. Esses motores necessitam de uma unidade eletrônica de controle para efetuar o controle da mistura ar/combustível, e também outros parâmetros que são configurados eletronicamente. Esse trabalho constitui em mostrar o desenvolvimento e gerenciamento eletrônico de motores ciclo Otto a partir de 6 ECUs (Eletronic Control Unit) em programação dos micros controladores Freescale e Microchip PIC, ambos em base da linguagem C e Assembly, em conjunto da FATEC Santo André e USP. A evolução parte do Microchip PIC até o Freescale, sendo esse o que possui todas as evoluções. Esse conjunto possui circuitos eletrônicos com as funções de condicionar sinais de entrada de sensores dentro do motor. Os parâmetros de entrada são processados pelo gerenciamento eletrônico, assim calculando a massa de combustível e avanço de ignição em todos os regimes do motor. Por fim a maior evolução no Microchip PIC é a Flex ECU que já conta com a tecnologia flex-fuel e na Freescale a tecnologia de cruise control.

\begin{abstract}
Currently internal combustion engines are widely used in passenger cars, motorcycles, transport vehicles and stationary equipment. These engines are in an electronic way of control for the control of the air / fuel mixture, and also like other parameters that are configured electronically. This is the joint work that shows the electronic assembly of machines from 6 ECUs (Electronic Control Unit) in programming of microcontrollers Freescale and Microchip PIC in conjunction with the language $\mathrm{C}$ and Assembly together of FATEC Santo André and USP. The same happens with the PIC Microchip evolving to a Freescale where it has all the evolutions. This set has electronic circuits with input signal conditioner functions that do not have a motor. Input parameters are processed by electronic control, as well as fuel mass and ignition advance at all engine speeds. Finally, the biggest evolution in the Microchip PIC is a Flex ECU that already counts on technology flex-fuel and Freescale to the cruise control.
\end{abstract}




\section{INTRODUÇÃO}

Com a expansão da produção de carros movidos a motores bicombustíveis e a preocupação cada vez maior com a emissão de gases, o Brasil vive uma demanda crescente por conhecimentos na área de eletrônica automotiva, especialmente em busca de soluções inovadoras no gerenciamento eletrônico de motores, a fim de reduzir a emissão de poluentes e melhorar a eficiência do motor [1].

A ECU utiliza diversos sensores para a aquisição de parâmetros físicos que definem mapas de funcionamento e estratégias para os atuadores, gerenciando o motor de forma a otimizar o consumo de combustível, emissões de poluentes e aumento de desempenho. As estratégias de software e hardware são mantidas em segredo pela indústria automotiva e seus fornecedores, dificultando a compreensão deste complexo sistema de gerenciamento [2].

A Escola Politécnica da USP e a FATEC Santo André em conjunto vem se dedicando ao desenvolvimento de unidades de gerenciamento eletrônico para motores a combustão interna, inicialmente com uma versão projetada e testada em mockup (motor estacionário, didático) com Microchip PIC (i. e. [3]). Após a segunda fase do projeto, tiveram duas frentes com Microchip PIC pela FATEC Santo André ainda utilizando o mockup (i. e. [4]), e começando a utilizar o Freescale pela Universidade de São Paulo para desenvolver uma ECU que fosse mais próxima as comerciais em um carro (i. e. [5]). Em 2014, a evolução das placas com PIC foi introduzida em um veículo comercial, sendo sua terceira geração (i. e. [6]). No ano seguinte foi introduzida uma nova placa com chip Freescale na sua segunda versão com aprimoramentos de hardware e software (i. e. [1]). E por fim, foi a versão Microchip IV em 2016 que foi introduzida a tecnologia flex (i. e. [2]).

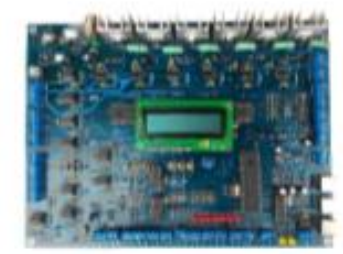

Microchip v. I 2011

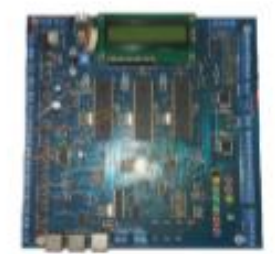

Microchip v. II 2013

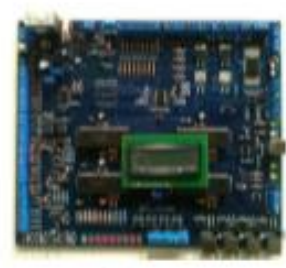

Microchip v. III 2014

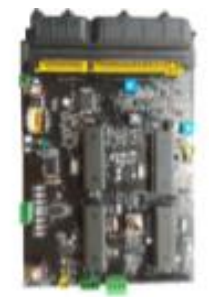

Microchip v. IV 2016

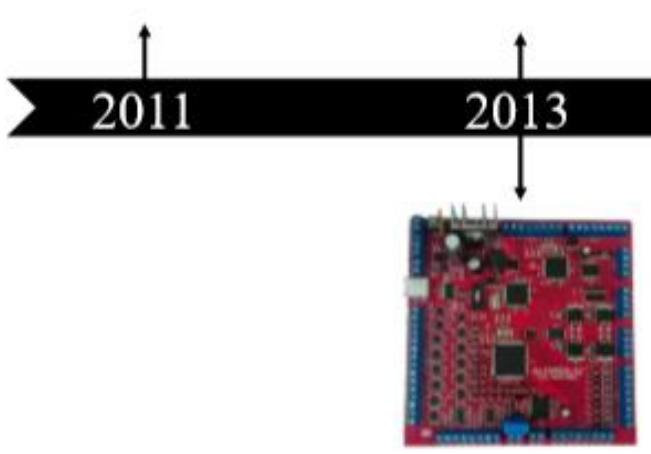

Freescale v. I 2013

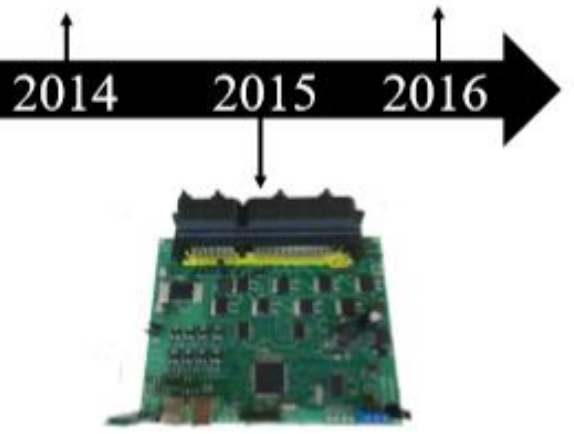

Freescale v. II 2015

Figura 1. Evolução das ECUs [Do autor]. 
O aperfeiçoamento desses módulos começou separadamente com alunos da FATEC Santo André em 2009, como exemplo de controle e condicionadores da válvula borboleta do motor pelo sistema drive-by-wire, e condicionadores de sinais de sensores do motor. Sua evolução chegou até a tecnologia flex e controle de injeção em malha fechada pela sonda lambda. Em paralelo, a USP foi desenvolvendo outra frente com chips utilizados em unidades que já existem nos mercados automotivos.

Com isto, espera-se com os desenvolvimentos destes trabalhos, viabilizar projetos futuros que busquem a inovação em áreas como injeção, ignição e gerenciamento do motor como um todo, bem como melhorias no controle do veículo, de forma a aperfeiçoar fatores como segurança, desempenho e conforto dos automóveis.

\section{Objetivos}

O objetivo principal deste trabalho é descrever as evoluções da ECUs do grupo de desenvolvimento de automotivo firmado entre a FATEC Santo André e Universidade de São Paulo.

As placas de desenvolvimento são constituídas de hardware, firmware e software, os sistemas eletrônicos fazem a correta leitura dos sensores presentes no motor e calcula, todos os parâmetros do motor, como tempos de abertura dos bicos injetores, sinais de ignição, controles de relés e posição da válvula borboleta, para garantir um controle estável da rotação do motor, na faixa de 800 RPM (marcha lenta) até 6000 RPM. Nos projetos iniciais tem a função do motor funcionar somente em marcha lenta, ou seja, parado sem torque exigido ao motor. A partir da versão 3 do Microchip PIC será empregado ao veículo, submetendo torques ao seu eixo. No caso das placas com Freescale, todas são testadas no veículo.

\section{Metodologia}

Foram estabelecidos vários desenvolvimentos da ECUs, em um processo evolutivo passando por várias versões de hardware e aprimoramento constante dos softwares, adicionando-se a cada etapa, novas funções. Portanto nesta primeira versão, são apresentadas condições mínimas para serem aplicadas em mockups e carros, com blocos básicos de softwares sem sofisticações, até a última com tecnologia flex e funções de aperfeiçoamentos de softwares complexos, similares às comerciais.

Assim os sistemas por tópicos foram descritos tendo em foco seus avanços, como o tipo de sistema utilizado em todas versões. 


\section{O início dos projetos: ECU Microchip PIC I}

\subsection{Visão geral}

Esse trabalho foi iniciado em 2009 com a adição de vários projetos paralelos até o seu final que é o mockup, ou seja, um motor doado pela montadora General Motor do Brasil (GMB) de 1.8L tipo estacionário que possui todos os equipamentos para controlar o motor. Entre esses equipamentos, o pedal de acelerador, todo seu sistema de chicotes e caixas de fusíveis e relés, tanque de combustível, sistema completo de arrefecimento, painel de instrumentos com todos os equipamentos em funcionamento e uma placa de ligações didática com todos os pontos de conexões no chicote, assim de realizar testes nos atuadores e sensores. O motor pode ser ligado e acelerado, mas sem atuação de torque no seu eixo.

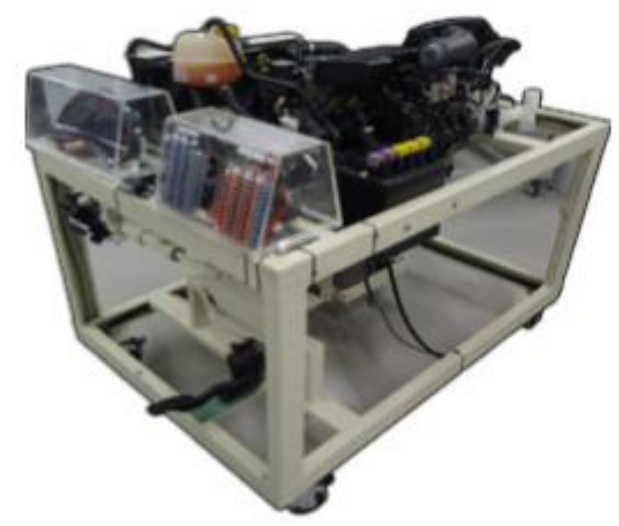

Figura 2. Mockup do projeto [3].

A função básica é um sistema didático, com a facilidade de ter a conexão à todos os pontos de sensores e atuadores externamente, sem necessidades de alterações na ECU ou chicote de motor/chassi/cluster.

\subsection{Descrição da ECU}

Nesta ECU, foi adotado o sistema descentralizado com blocos de gerenciamento, sincronismo e comunicação separados por três Microchips PIC. Conforme ilustrado na Figura 3, os blocos de comunicação, de gerenciamento e de sincronismo são feitos por protocolo SPI, e o bloco de gerenciamento para a comunicação é via USART. 


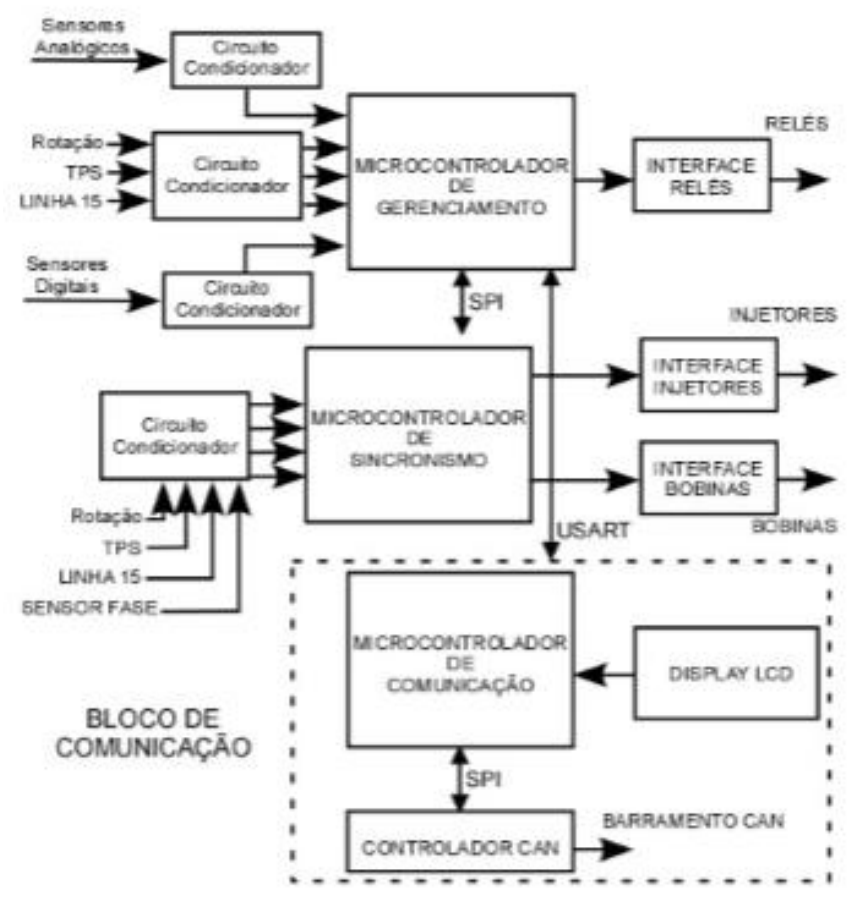

Figura 3. Diagrama de blocos da ECU [3].

O hardware referente ao bloco de gerenciamento é constituído de circuitos de condicionamento para os sinais analógicos e digitais, circuito de condicionamento do sinal proveniente da roda fônica (sensor do veículo é indutivo), microcontrolador PIC 18F452 e interface de controle da válvula borboleta e todos os sensores de aquisição [3].

O bloco de sincronismo ilustrado na Figura 4 é constituído de outro microcontrolador PIC18F452, transistores para acionamento dos relés e circuitos de interfaces para o acionamento das válvulas injetoras de combustível e bobinas de ignição [3].

O bloco de comunicação e diagnose é constituído de mais um microcontrolador PIC18F452, um display de LCD 16X2 e circuito controlador CAN com o seu respectivo receiver, conforme Figura 5.

\subsection{Circuitos condicionadores e atuadores}

O PWM foi controlado conforme a solicitação do motorista (pedal do acelerador) e rotação do motor (limitando a abertura e fechamento para que o motor não admita mais ar que o necessário ou que reduza demais a desaceleração). O CI Freescale MC33186 (ponte H) foi utilizado para o controle do motor da válvula borboleta. Outro atuador utilizado foi o transistor como chave para comandar relés do chicote de motor.

O circuito integrado utilizado para condicionamento do sinal da roda dentada foi o LM1815 que tem a função de obter o sinal em forma senoidal e na sua saída gerando onda quadrada. Outros amplificadores de sinais com filtros na sua entrada (capacitores) foram utilizados para aquisição dos sensores analógicos.

O módulo LM358 possui amplificadores operacionais LM358 configuradas como seguidor de tensão (buffer), que tem a função de isolar a impedância do sensor do resto do circuito da ECU, e outro amplificador operacional (LM358) configurado como filtro ativo 
de segunda ordem na topologia sallen key tipo passa baixas. Sensores analógicos de 0 a $5 \mathrm{~V}$ foram constituídos das seguintes formas descritas na Tabela 1, e na questão dos sensores digitais, a configuração está descrita na Tabela 2.

Tabela 1. Sensores analógicos da ECU [3].

\begin{tabular}{cc}
\hline Sensor & Bloco de destino \\
\hline Posição da borboleta (TPS) & Gerenciamento e Sincronismo \\
Posição do pedal & Gerenciamento e Sincronismo \\
Pressão do coletor de admissão (MAP) & Gerenciamento \\
Sensor de detonação & Gerenciamento \\
Temperatura do Ar & Gerenciamento \\
Temperatura da água & Gerenciamento \\
Sonda Lambda & Gerenciamento \\
Reserva & Sincronismo \\
\hline
\end{tabular}

Tabela 2. Sensores digitais [3].

\begin{tabular}{cc}
\hline Sensor & Bloco de destino \\
\hline Linha 15 (ignição) & Gerenciamento \\
Sensor de velocidade & Gerenciamento \\
Interruptor do freio & Gerenciamento \\
Interruptor de embreagem & Gerenciamento \\
Linha 15 & Sincronismo \\
Sensor de fase & Sincronismo \\
Reserva 1 & Sincronismo \\
Reserva 2 & Sincronismo \\
\hline
\end{tabular}

Com a utilização de amplificador LM1949 e uma configuração do circuito em Darlington foi utilizada para a o chaveamento das boninas de ignição e bicos injetores.

\subsection{Interface de comunicação CAN}

Utiliza-se um controlador CAN (MCP2515), um transmissor CAN (PCA82C251). O bloco de comunicação comunicará com o bloco de gerenciamento através de um protocolo USART e com o controlador CAN através de um protocolo SPI.

\subsection{Resultados e conclusão}

Os resultados obtidos neste projeto foram positivos em relação à escolha de uma metodologia didática, permitindo que os alunos da Fatec Santo André e da Escola Politécnica da USP utilizem os conhecimentos adquiridos com este projeto no desenvolvimento de novos trabalhos.

Também com os resultados obtidos em experimentos seccionados com elementos reais antecipam que com o aperfeiçoamento dos softwares, seguramente será possível gerenciar o motor GM 1.8 do mockup, considerando-se que todas as entradas e saídas da ECU original foram previstas neste trabalho. 
A experiência adquirida no desenvolvimento desta primeira versão criou uma dinâmica de trabalho e soluções que abreviará significativamente o desenvolvimento de novas versões [3]. Abaixo mostra a confecção da ECU do projeto, com todos os respectivos blocos e sistemas de condicionadores (i e. [3]).

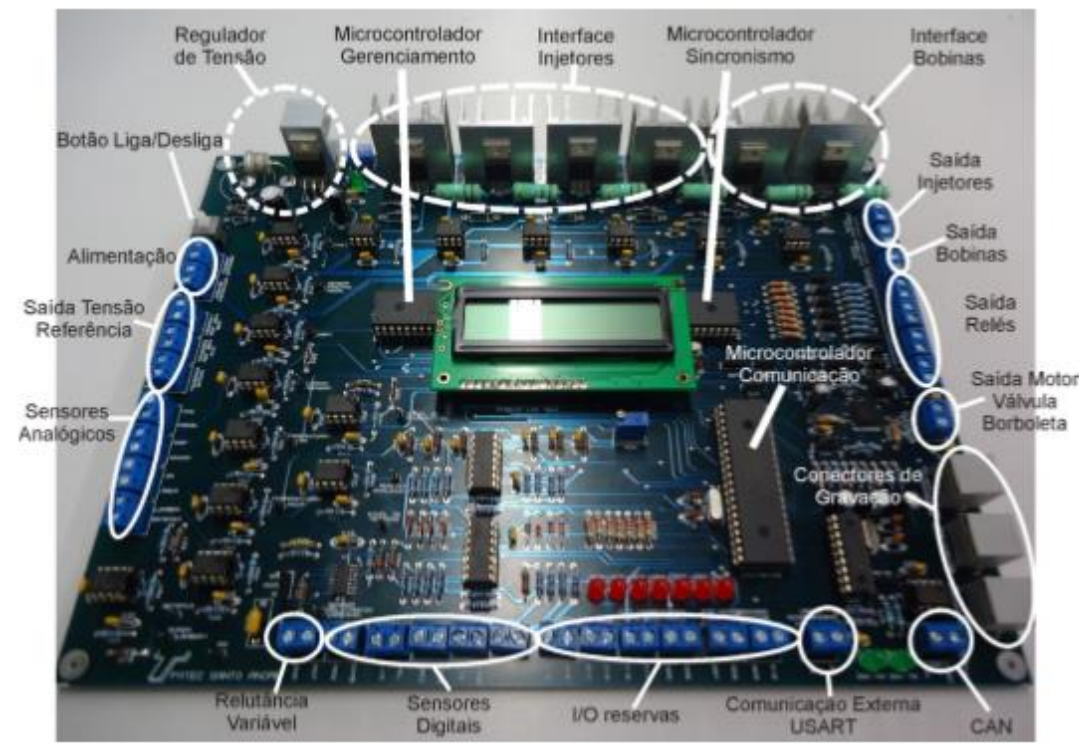

Figura 4. ECU PIC versão I [3].

\section{Segundo desenvolvimento: ECU Microchip PIC II}

\subsection{Visão geral}

Nesta segunda geração com Microchip PIC, a unidade foi aperfeiçoada em alguns pontos, tais como: novo layout da placa, com nova organização dos componentes e correções eletrônicas. Com o presente trabalho anterior, foi possível uma comparação entre a ECU original e atual, assim resolvendo problemas principais que não tinham uma boa resposta do motor comparados as características originais, isso devido o projeto realizar o funcionamento do motor.

O desenvolvimento do hardware foi realizado em duas fases: na primeira, foi fabricada uma placa de circuito impresso empregando circuitos integrados mais didáticos, já existentes no mercado, para a implementação das interfaces de condicionamento dos sinais proveniente dos diversos sensores, assim como para as interfaces de controle das válvulas injetoras e bobinas de ignição, com a intenção de familiarizar-se com o controle e o funcionamento de um gerenciamento eletrônico do motor.

$\mathrm{Na}$ segunda fase foram utilizados circuitos integrados de interfaces fabricadas pela Freescale tanto para os sensores como para os atuadores, tendo em vista reduzir o tamanho da placa, aumentar a confiabilidade e facilitar as ações de diagnose, uma vez que essas interfaces fornecem parâmetros (ex: corrente de carga) de diagnóstico das válvulas injetoras e bobinas de ignição. As duas placas estão compostas por circuitos específicos como o de enquadramento do sinal proveniente da roda dentada geradora de impulsos, interface para a linha CAN (Controller Area Network) e ponte-H para controle da válvula borboleta. O driver de Injeção e Ignição corresponde ao CI 33810 da Freescale, e possui diversos modos 
de funcionamento, cuja configuração é feita pelo protocolo SPI (configurado pelo PIC gerenciamento). No primeiro caso, o transistor que aciona a bobina de ignição está presente na placa. Neste caso, o CI pode medir e controlar parâmetros da ignição, como a corrente do primário da bobina ou a duração da centelha.

Como unidade de processamento em ambas as placas, foram utilizados três microcontroladores PIC18F452 da Microchip, descentralizando a unidade de gerenciamento em três blocos: gerenciamento, sincronismo e comunicação / diagnose. Este trabalho limitou-se em desenvolver os softwares de controle para os blocos de gerenciamento e sincronismo permitindo assim que outros trabalhos surgissem no controle do bloco de diagnóstico/comunicação.

No bloco de gerenciamento, o software de controle tem a função de atuar em alguns componentes básicos do motor (relés, válvula borboleta e etc.), monitorar os sensores (rotação, pressão, temperatura do ar admitido, pedal e posição da válvula borboleta - TPS), calcular e definir parâmetros de controle (tempo de injeção, tempo de carregamento da bobina de ignição, etc.) e transmitir todos esses parâmetros para o bloco de sincronismo. Por sua vez, o software do bloco de sincronismo é responsável por receber os parâmetros enviados pelo gerenciamento, armazenar esses dados em uma matriz localizada na memória de programa do microcontrolador e, através do sinal da roda dentada, sincronizar os sinais de comando do sistema de injeção e ignição do motor. Assim, é possível obter um controle do motor em diversos regimes de funcionamento do mesmo, permitindo futuros desenvolvimentos e aplicações nessa área de pesquisa [4]. A Figura 5 mostra como ficou a placa de circuito impresso com seu respectivo layout:

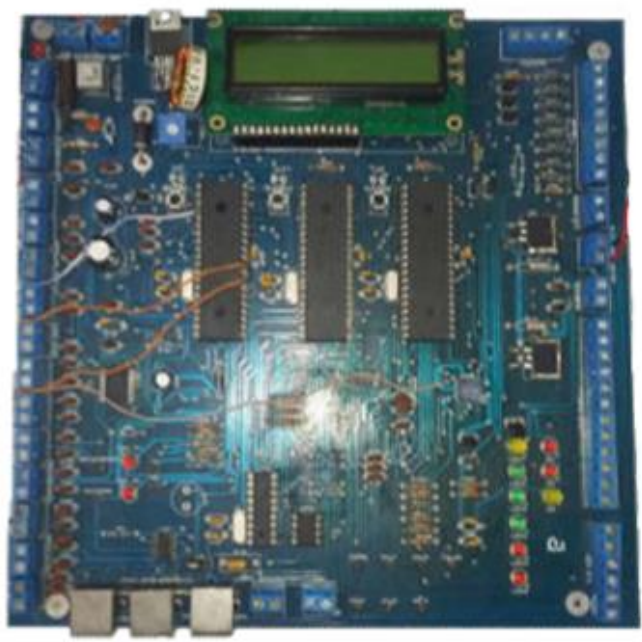

Figura 5. ECU PIC versão II [4].

\section{Primeira versão: ECU v2.0 Freescale}

\subsection{Objetivo}

Tem-se como principal objetivo desta ECU o desenvolvimento de uma unidade de gerenciamento eletrônico para um motor a combustão interna modelo Volkswagen 2.0L 8V, aplicado a um veículo modelo Polo Sedan 2004, substituindo integralmente a unidade original do veículo [5]. 


\subsection{Plataforma do projeto}

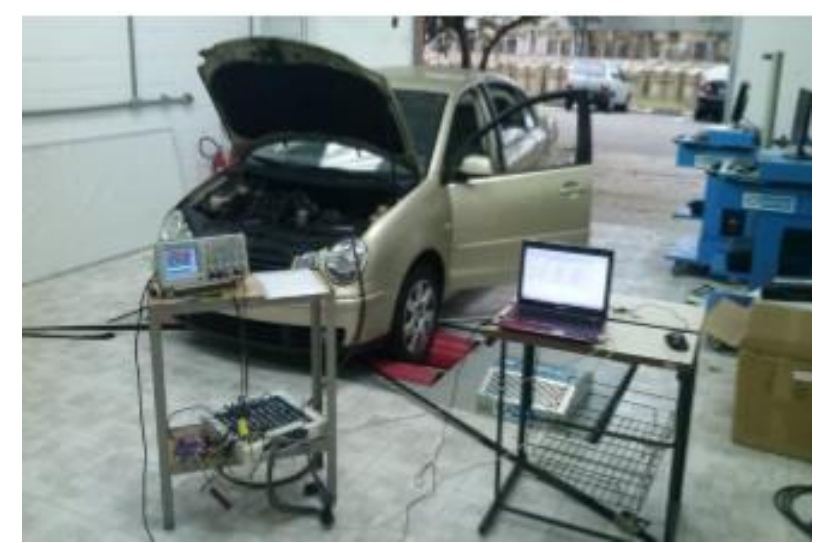

Figura 6. Plataforma do projeto para o desenvolvimento do software [5].

A plataforma de projeto consiste de um veículo modelo polo aplicado em um dinamômetro inercial, com objetivo de desenvolver a estratégia do firmware e avaliar o desempenho do motor em condições de carga [5].

\subsection{Descrição da ECU}

No diagrama da figura acima destacam-se os três principais blocos do sistema (Gerenciamento, Sincronismo e Comunicação), caracterizando uma arquitetura descentralizada com três unidades de processamento [5]. As tarefas executadas por cada bloco foram resumidas a seguir:

- Gerenciamento: Leitura de sensores e cálculo dos parâmetros de atuação para injeção, ignição, válvula borboleta e relés;

- Sincronismo: Geração dos comandos de atuação para injeção e ignição (com parâmetros recebidos do Gerenciamento via protocolo SPI);

- Comunicação: Coleta periódica de dados dos blocos de Gerenciamento e Sincronismo, e envio dos mesmos para um computador externo (via protocolo USB) e para o painel do motorista do veículo (via protocolo CAN), além da leitura de velocidade do veículo (via protocolo CAN) [5].

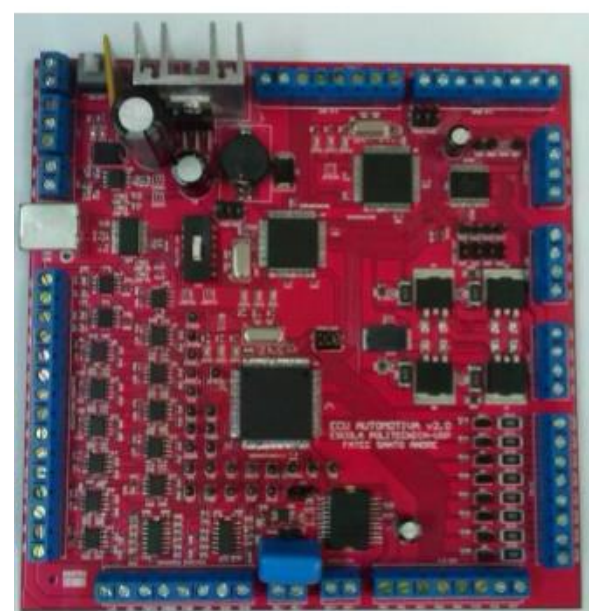

Figura 7. Protótipo da ECU v2.0. 
Dentre as principais características do hardware que foram evoluídos:

- Emprego de microcontroladores automotivos Freescale da família S12XE, formada por microcontroladores projetados para aplicações de gerenciamento de motores automotivos;

- Uso de filtros analógicos e comparadores de tensão para o tratamento de sensores analógicos e digitais;

- Emprego de circuitos de proteção contra sobretensão e curto-circuito;

- Suporte à comunicação USB 2.0 com um computador externo;

- Emprego de um circuito integrado (CI) mais robusto para tratar o sinal de rotação proveniente do sensor de relutância variável, evitando problemas com ruídos [5].

\subsection{Evoluções de software}

\subsubsection{Bloco de gerenciamento}

O microcontrolador de Gerenciamento é responsável pelo cálculo dos parâmetros de atuação do sistema, tomando como base os valores dos sensores presentes no motor. $\mathrm{O}$ processamento ocorre de maneira a garantir um controle de rotação estável. Sendo assim, modelou-se o controle de rotação para posterior implementação no microcontrolador ( $\mathrm{uC}$ ) de Gerenciamento [5].

O controle de posição da válvula borboleta foi implementado com um controlador PI (proporcional e integral), sendo que a referência de posição para este controlador é determinada pelo controle de rotação que atua simultaneamente no tempo de injeção de combustível e na abertura da válvula borboleta, responsável pelo controle do fluxo de ar admitido pelo motor. Uma parte do controle do tempo de injeção consiste de um controlador puramente proporcional $(\mathrm{P})$, cuja finalidade é enriquecer a mistura a/c quando a referência de rotação for maior que a rotação medida.

Além da borboleta, o controle de rotação necessita que a injeção de combustível esteja funcionando corretamente. Para a determinação do tempo de injeção, seguem-se os passos abaixo:

- Cálculo da rotação do motor;

- Estimativa da massa de ar admitida por cilindro com base na equação de estado dos gases ideais (ou seja, adotou-se que o ar se comporta como um gás ideal);

- Cálculo da massa de combustível a ser injetada de acordo com a relação estequiométrica de 13,3g de ar para $1 \mathrm{~g}$ de gasolina com $22 \%$ de etanol);

- Cálculo do tempo de injeção, com base no fluxo do bico injetor;

- Aplicação de um fator multiplicativo (determinado de forma empírica), com base na aceleração e na temperatura da água [5].

\subsubsection{Bloco de sincronismo}

O firmware do uC de Sincronismo contém a estratégia para acionar precisamente os sinais de injeção e ignição. Para isto, este microcontrolador utiliza os parâmetros de injeção e ignição calculados pelo uC de Gerenciamento. 
Analogamente ao Gerenciamento, o Sincronismo deve manter sincronia com o sinal de rotação, proveniente da associação da roda fônica com o sensor de relutância variável. Para isto, o Sincronismo deve detectar a falha (correspondente à ausência de dois dentes) da roda fônica, a fim de reiniciar contagens e atualizar parâmetros para a próxima volta e acionar os bicos injetores e a bobina de ignição [5]. O sensor de fase determina a correta sequencia de injeção desde a partida.

\subsubsection{Bloco de comunicação}

O uC de Comunicação é, de modo geral, responsável por estabelecer a conexão entre a ECU e outros dispositivos externos, como computadores, aparelhos automotivos de diagnose ou até outras ECUs presentes no veículo (como, por exemplo, a ECU do painel do motorista). Deste modo, o uC de Comunicação pode ser visto como uma verdadeira ponte que interliga a ECU deste trabalho ao meio externo [5].

Para se comunicar com a aplicação (software) de monitoramento, o bloco de Comunicação utiliza o protocolo UART, dado que este é um protocolo de fácil integração no software e possui suporte no ambiente Windows [5].
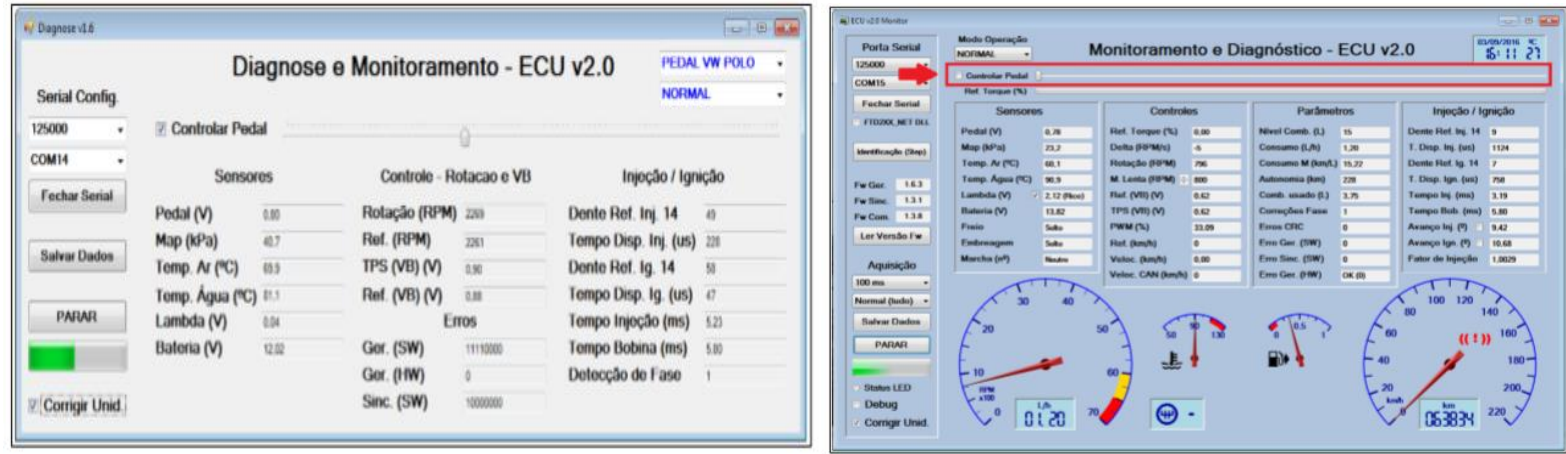

Figura 8. Evolução da interface de aquisição [5].

\subsection{Resultados}

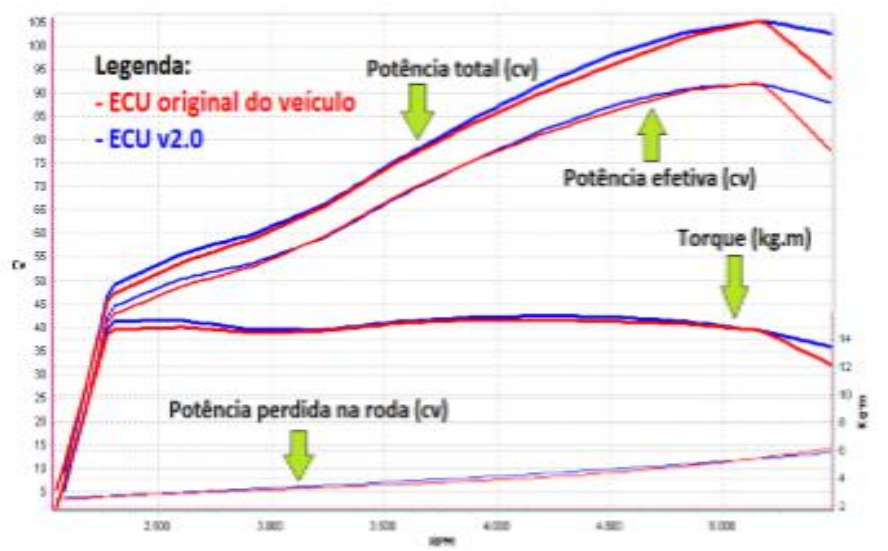

Figura 9. Curva de potência e torque da ECU original e da ECU v2.0 [5].

Os valores da ECU original e o trabalho proposto mostraram-se muito semelhante, conforme constatado pelo gráfico na Figura 9. O trabalho cumpriu com a sua meta inicial de desenvolver uma unidade de gerenciamento eletrônico para um motor Volkswagen 2.0L, operado inclusive em condições de carga. O trabalho demonstrou que é possível se controlar 
um motor à combustão interna por meio de um controle de rotação, responsável também pelo fornecimento de torque ao eixo do motor em condições de carga.

Pelos resultados obtidos é possível notar que, com a implementação do controle de rotação, a ECU deste trabalho responde semelhantemente a ECU original do veículo, incluindo a característica de filtragem do pedal de aceleração, de modo a suavizar acelerações bruscas quando o veículo se encontra em movimento. Além disto, é importante ressaltar que a unidade eletrônica desenvolvida neste trabalho atua no motor de forma totalmente independente e sem qualquer auxílio da ECU original (relembrando que a ECU original foi completamente desligada do motor).

\subsection{Evolução da ECU Freescale I}

Foi desenvolvido um controlador de cruzeiro para operar no mesmo veículo utilizado na versão I. Também terá o intuito de melhorar o funcionamento da unidade de gerenciamento, para preparar a implementação deste novo controlador.

Essa segunda evolução da ECU Freescale foi realizada em 2017 para os aprimoramentos estão nas diversas malhas como: controle de marcha lenta, controle de posição da borboleta eletrônica e controle de partida. Foi implementado controle de torque, controle de mistura ar/combustível em malha fechada através da sonda lambda, segurança na comunicação entre blocos da unidade de controle eletrônico, compensação de parâmetros de injeção e ignição, identificação da marcha engatada, suporte para diagnóstico do sistema via protocolo OBDII.

A substituição do controle de rotação pelo controle de torque em malha aberta, sendo comente em marcha lenta. A reformulação da ECU, terá uma melhora significativa na dirigibilidade do veículo.

Além dessas atividades descritas, tem um novo desenvolvimento na parte de aquisição em tempo real, outras capacidades foram adquiridas como: monitorar outros componentes do carro, não pertinentes ao motor, função de computador de bordo função de tipos de utilização do teste.

\section{Terceira versão: Microchip PIC III}

\subsection{Objetivo}

Este trabalho está baseado em uma rotina de programação para controle de um motor a combustão ciclo Otto, atualmente em linguagem $\mathrm{C}$ de microcontrolador Freescale sendo convertido para o código semelhante à programação em PIC, sendo a principal diferença: as rotinas das funções de cada microcontrolador.

O objetivo principal deste estudo, o desenvolvimento de uma ECU em Microchip PIC de baixo custo com características similares a ECU desenvolvida pela POLI-USP/FATEC Santo André com microcontrolador Freescale que possui características de hardware com desempenho superior devido à sua tecnologia mais recente. 


\subsection{Descrição geral}

A partir dos conhecimentos adquiridos nos projetos ciclo Otto II (i. e. [4]) e Unidade de Gerenciamento Eletrônico de um Motor Volkswagen 2.0L: Projeto Otto II (i. e. [5]) e projetos anteriores nesta plataforma de microcontrolador PIC, desenvolvemos as condições similares de funcionamento da ECU confeccionada em Freescale para esta plataforma. Foi utilizado o mesmo veículo da ECU Freescale v2.0, o Volkswagen Polo 2.0L 2004 para a realização dos testes e condicionamento da ECU para o motor.

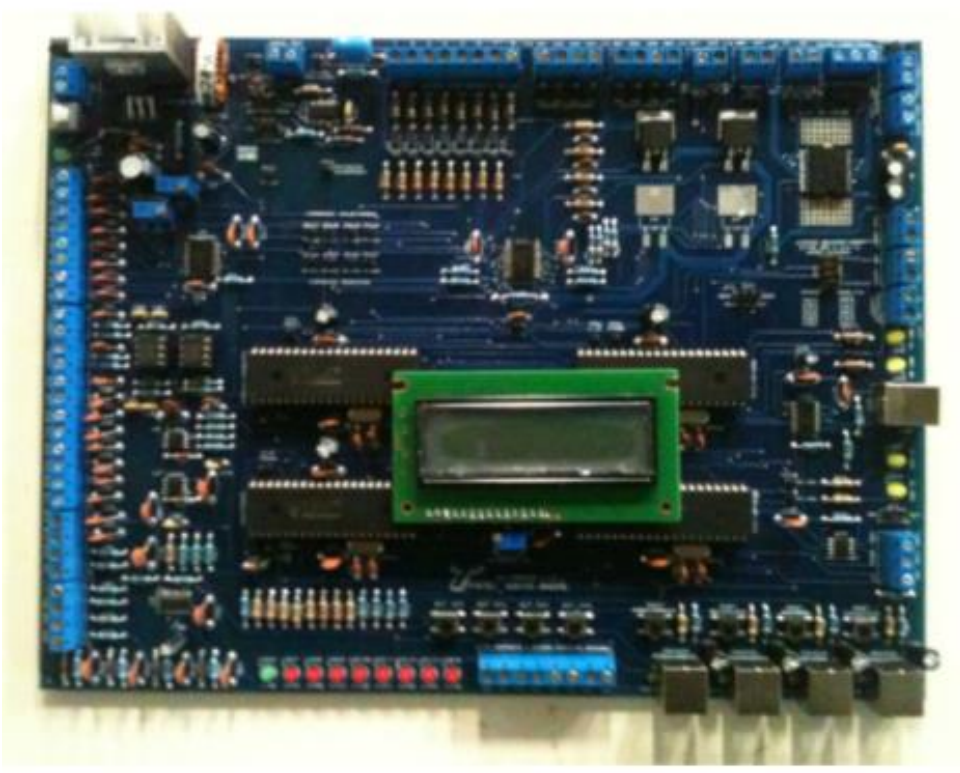

Figura 10. ECU com microcontrolador PIC V1.2 [6].

\subsection{Hardware}

No diagrama da ECU II Microchip PIC se destacam os quatro principais blocos do sistema (gerenciamento, sincronismo, admissão e comunicação), caracterizando uma arquitetura descentralizada com quatro unidades de processamento. Nota-se ainda o grande fluxo de dados entre os processadores (seguindo uma comunicação via protocolo SPI), o que é uma característica marcante da arquitetura descentralizada.

As tarefas executadas por cada bloco foram resumidas a seguir:

- Gerenciamento: Leitura de sensores do veículo, cálculo dos parâmetros de atuação para injeção, ignição, válvula borboleta e acionamento do relé da bomba de combustível;

- Sincronismo: Geração dos comandos de atuação para injeção e ignição (com parâmetros recebidos do Gerenciamento via protocolo SPI);

- Admissão: Leitura do sensor de TPS e Pedal, cálculo dos parâmetros geração do comando (pulsos PWM) de atuação para a válvula borboleta (drive-by-wire);

- Comunicação: Coleta periódica de dados dos blocos de Gerenciamento e Sincronismo, e envio dos mesmos para um computador externo (via protocolo USB) e para o painel do motorista do veículo (via protocolo CAN) [6]. 
Com relação ao hardware deste projeto, diversas mudanças e aperfeiçoamentos foram conduzidos, de forma a melhorar o desempenho do sistema. Dentre as principais mudanças, destacam-se:

- Mudança no microcontrolador de sincronismo do $18 \mathrm{~F} 452$ para o $18 \mathrm{~F} 44 \mathrm{~K} 22$, pois possui uma maior quantidade de temporizadores de rotinas de programação, assim dedicando um timer para cada injetor e 2 para cada bobina (duas bobinas, funcionamento por centelha perdida);

- Adição de uma comunicação USB 2.0 com um computador externo (diagnose para leitura de valores - tempo injeção, ignição, sensores analógicos);

- Adicionados pinos de medição de todos os sinais de sensores e atuadores;

- Adição do chip de Admissão (18F452), pois o chip de gerenciamento está tratando e realizando muitos cálculos e assim não possuindo mais temporizadores para a atuação da válvula borboleta, para não sobrecarregar o processamento deste módulo;

- Sensores MAP, Temperatura de Ar e água modificados do CI MC33975 para as portas $\mathrm{AD}$ (analógicas) do bloco de gerenciamento - portas AN0, AN1 e AN2, alterado via hardware;

- Alterado LEDS da placa para indicar o acionamento dos injetores e bobinas LED 2 do PIC Gerenciamento para injetor 1, LEDs 1 e 2 do PIC Sincronismo para injetores 2 e 3, LEDs 1 e 2 do PIC de admissão para injetor 4 e bobina 1 e por fim LED 1 do PIC de comunicação para a Bobina 2.

- Utilizando circuitos externos de condicionamento do sinal para sensor MAP, temperatura de água e temperatura do ar, devido ao MUX CI33972 sobrecarregar a comunicação SPI [6].

\subsection{Software}

Os mesmos cálculos foram utilizados do projeto em Freescale (Pereira, 2013) somente realizados algumas mudanças para que o microcontrolador utilizado (Microchip PIC) suportasse esse processamento. Sendo assim, os códigos dos microcontroladores de cada bloco do sistema de ambos os projetos são bastante semelhantes. Por ser uma unidade eletrônica com base maior em cálculos, o controle deste projeto se mostrou bastante eficiente e estável, já demonstrado no projeto com o microcontrolador Freescale. Somente foram realizadas alterações em alguns cálculos de modo que eles atenderiam as características de configuração do Microchip PIC [7]. O mesmo software de aquisição da ECU Freescale (i. e. [5]) foi utilizado neste sistema.

\subsection{Resultados do projeto}

Para efeito de comparação realizamos o teste de potência do motor no dinamômetro inercial que possuímos em nossa faculdade com a ECU original que apresentou valores descritos abaixo: 

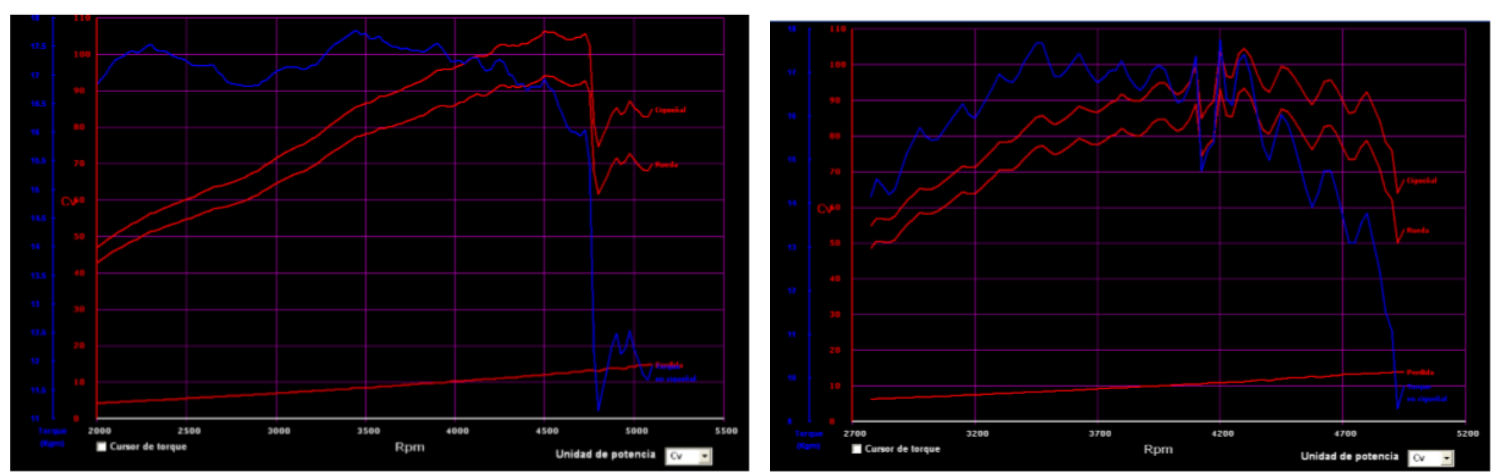

Figura 11. Comparação da ECU original com a ECU Microchip PIC III [7].

O gráfico apresenta três informações, sendo no eixo " $x$ " a rotação e no eixo " $y$ " o torque (em azul) e a potência (em vermelho). Como mostrado acima pela curva de potência com a ECU $1.2 \mathrm{~V}$, verifica-se uma instabilidade no sinal devido à oscilação da válvula borboleta, que poderá ter um controle mais refinado em projetos posteriores.

A partir de 3500 RPM existe uma leve queda de potência devido ao cruzamento dos pulsos dos temporizadores de injeção e ignição, resolvida parcialmente com ajustes da programação, cuja solução também precisa ser revista futuramente.

\section{Freescale II}

\subsection{Objetivo}

Esse módulo teve na sua segunda versão a modificação da placa com o microcontrolador Freescale, com correções e acréscimos de todas evoluções das ECU anteriores e principalmente a tecnologia bicombustível (etanol e gasolina).

\subsection{Aprimoramentos}

A parte de condicionadores analógicos e digitais são iguais última versão do PIC, ou seja, modelo III. Foi inserido comunicação bluetooth, ainda não ativada. Utilizando somente um processador para todo o controle do motor, no caso se tornou de uma arquitetura descentralizada para centralizada (único microcontrolador para processamento). 


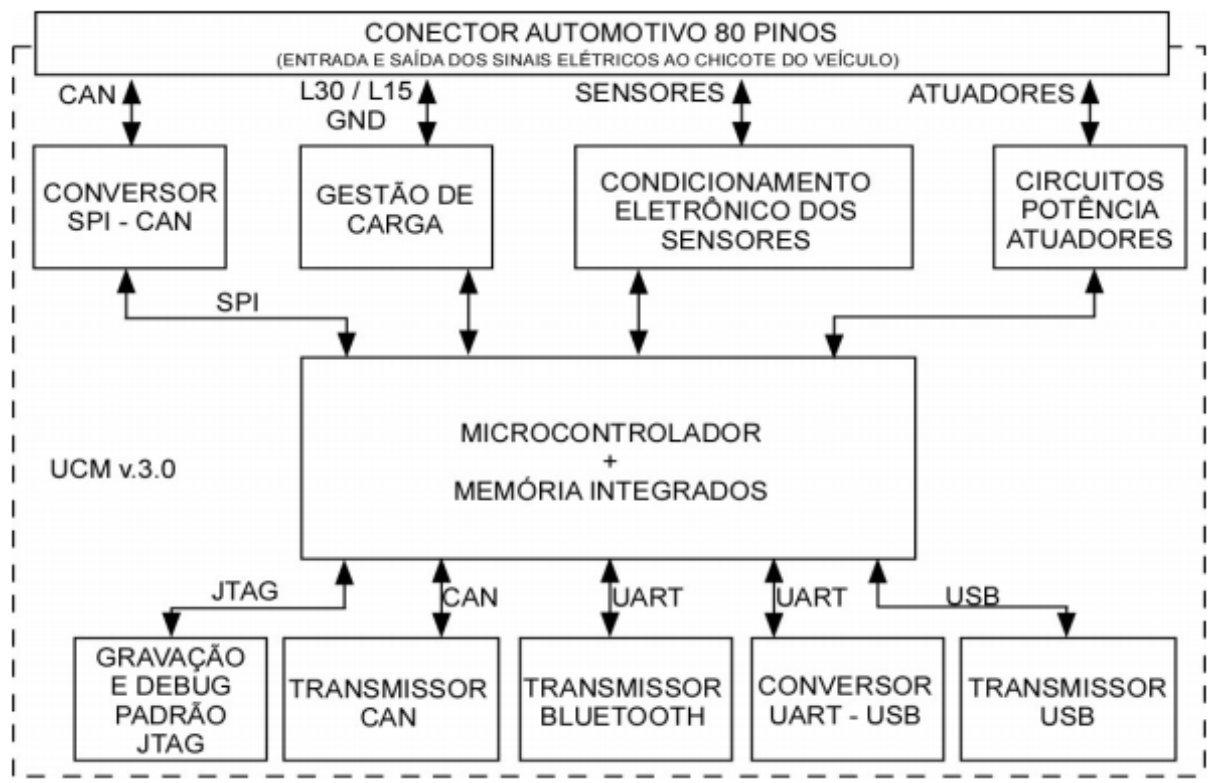

Figura 12. Diagrama ECU Freescale II [8].

As especificações do projeto do hardware usaram uma concepção centralizada, neste caso apenas um microcontrolador para gerenciar todas as funções da UCM. Tendo em vista a complexidade existente em um gerenciamento eletrônico do motor e a quantidade de sensores $\mathrm{e}$ atuadores a serem analisados e processados, $\mathrm{o}$ microcontrolador escolhido é da família K40, modelo MK40X256ZLQ100, fabricado pela Freescale. Este possui núcleo ARM CORTEX M4 de 32bits com possibilidade de instruções DSP, com alto desempenho e com baixo consumo de energia.

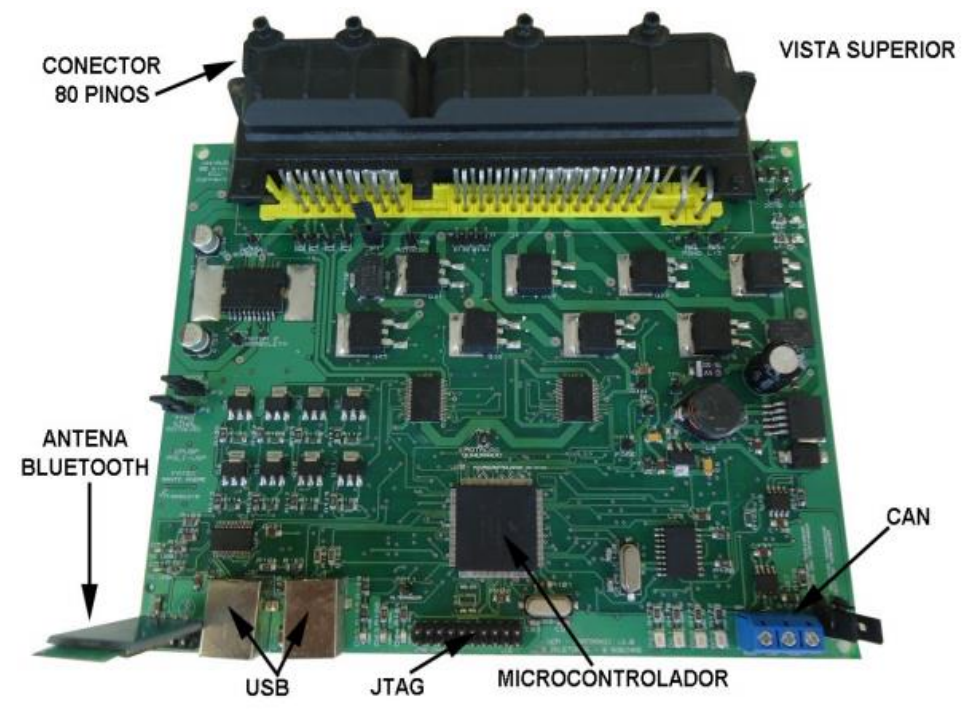

Figura 13. ECU Freescale II [8].

Como o desenvolvimento do algoritmo de gerenciamento foi muito longo optou-se a utilizar aplicações automotivas em tempo real, integradas ao sistema. O ideal nesta aplicação seria utilizar AUTOSAR (Automotive Open System Architecture), que é na arquitetura de firmware automotivo padronizado. O objetivo do AUTOSAR é criar e estabelecer padrões para sistemas automotivos, que irá proporcionar uma infraestrutura básica para ajudar com o desenvolvimento do firmware. Entretanto o RTOS (REALTime Operating System), que possui algumas aplicações com licenças gratuitas que 
compatíveis com o microcontrolador escolhido, acabou sendo escolhido. Ele também apresenta excelentes resultados em testes práticos, se mostrando eficaz para atender a toda estrutura de firmware proposta. Um RTOS facilita a divisão do trabalho entre interrupções e tarefas, melhorando o desempenho e diminuindo consideravelmente tempo de latência do sistema. Com o RTOS, a arquitetura do formato pode ser toda baseada em eventos e interrupções, economizando processamento e melhorando a eficiência da aplicação [8].

\subsection{Resultados}

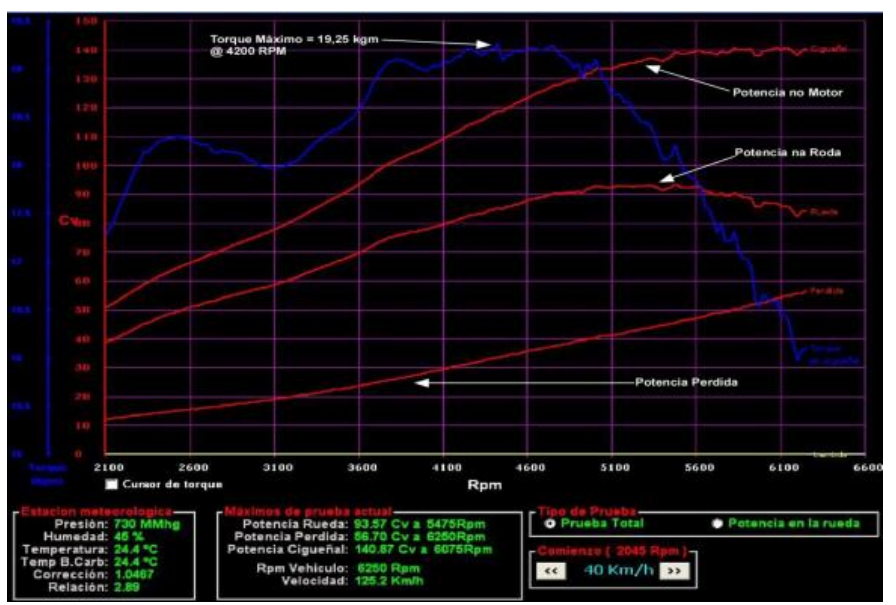

Figura 14. Medições finais do dinamômetro inercial [8].

Verificando neste teste a potência obtida na roda foi de $93,57 \mathrm{Cv}$ a $5475 \mathrm{rpm}$, foi optado por linearizar ao máximo a curva final do motor. Antes desse teste, foram realizados outros testes, variando o ganho proporcional e integral do controlador de rotação objetivando de aumentar a potência máxima na roda e aproximar dos valores originais do carro. No entanto só obteve 100 cavalos de potência, pois apareceram instabilidades nos valores finais, e então foram adotados parâmetros conservadores de baixa potência e torque.

\section{Quarta versão: Microchip PIC IV}

\subsection{Objetivo}

O presente projeto consiste em desenvolver uma ECU a partir dos projetos anteriores (i.e. [5]; i. e. [7]), utilizando microcontroladores da família Microchip PIC para gerenciar o motor de um veículo Volkswagen GOL 1.6L (EA111) flex 2009. O projeto está divido em duas etapas sendo o aperfeiçoamento do hardware apresentado por (i. e. [7]) para uma placa de menor dimensão, com maior poder de processamento, adição de novos drivers, como sensor lambda e sensor de detonação, e possibilidade de implementação de novas funcionalidades em futuros projetos, bem como e a melhoria do software para um programa estruturado em módulos. 


\subsection{Visão geral}

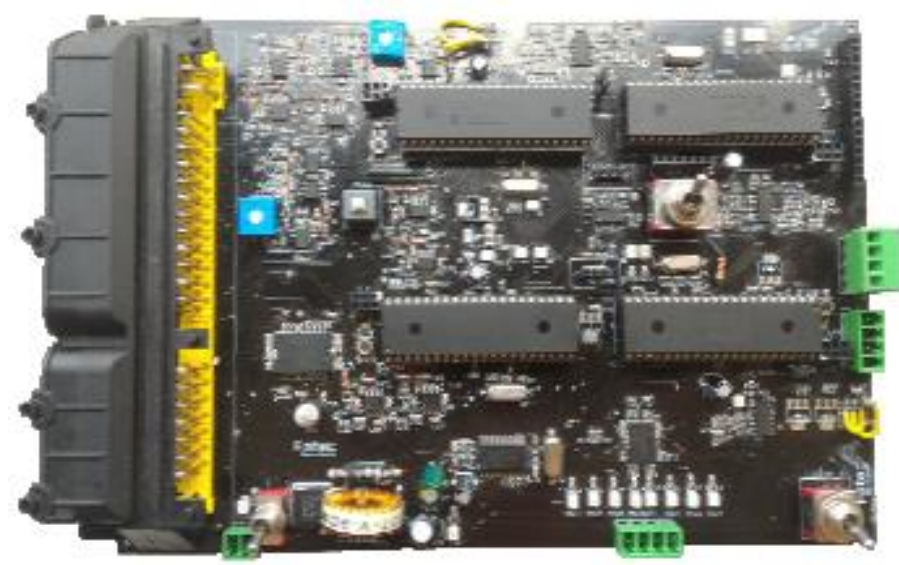

Figura 15. ECU versão IV com Microchip PIC [2].

Mesmo princípio da ECU de versão anterior possui 4 microcontroladores, mas foi adicionado condicionadores de sinais de sensores de detonação e aquisição para sensor lambda para realizar o controle de malha fechada e também o uso de um conector automotivo para que fosse possível conectar a ECU diretamente no veículo, da mesma forma que a ECU original. No bloco de comunicação foi acrescentado o módulo bluetooth. A interface via computador foi realizada, mas com modificações de estética para o adequar ao projeto atual.

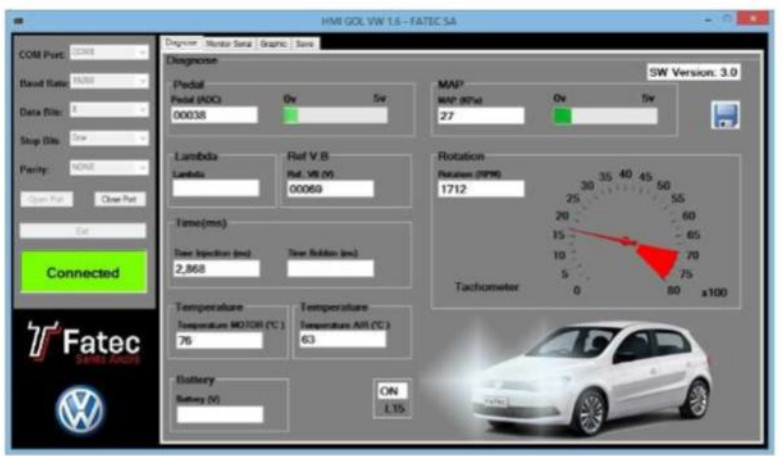

Figura 16. Atualização na interface [2].

\subsection{Resultados}

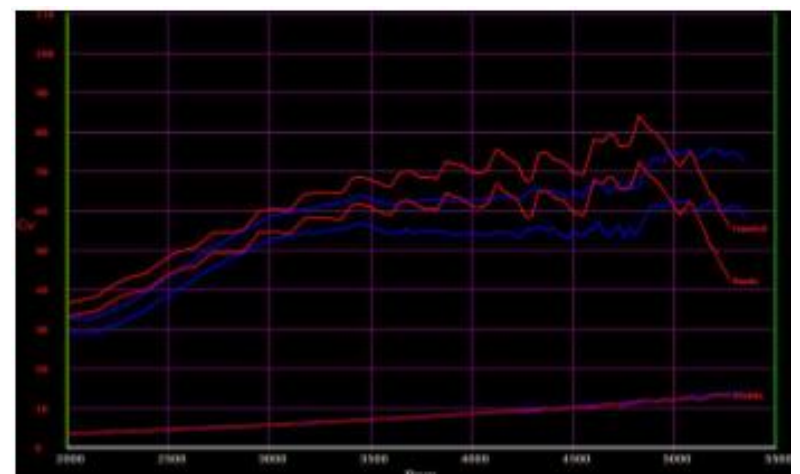

Figura 17. Comparativo das curvas de potência e torque da ECU original e a ECU Microchip PIC IV [2]. 
O gráfico apresenta três informações sendo no eixo " $x$ " a rotação e no eixo " $y$ " o torque (em azul) e a potência (em vermelho).

Concluído que o hardware funcionou de forma satisfatória, apesar de alguns erros no layout da placa. O software foi otimizado, reduzindo o tempo de processamento de $12,50 \mathrm{~ms}$ para $5,40 \mathrm{~ms}$, mantendo-se a mesma estratégia do projeto anterior (i.e. [7]), apresentando um resultado satisfatório. O levantamento de torque e potência mostrou que a ECU desenvolvida neste projeto chegou próxima de alcançar o mesmo desempenho do original, porém, ainda é preciso realizar mais calibrações e agregar funções para otimizar a curva de torque e potência [2].

\section{CONCLUSÃO}

Verificamos nesse artigo todas as evoluções e atualizações das ECUs do grupo de eletrônica automotiva das instituições FATEC Santo André e Universidade de São Paulo. Alunos das FATECs Sorocaba e Taubaté também estão envolvidos nestes projetos, iniciando os projetos também em outros veículos. Mostrou-se que foi adquirido conhecimentos avançados na área de gerenciamento de motores de combustão interna, tendo como frentes dois tipos de microcontroladores, um com suas aplicações já ingressada na área automotiva e outro com aplicações de diversas áreas da eletrônica e automação, servindo também como base didática. O controle dos motores a partir destas ECUs estão sendo cada vez mais aprimorados, tendo em vista que o desempenho está muito próximo das características das ECUs originais, portanto com os projetos em andamento e futuros certamente teremos evoluções cada vez mais próximas à das ECUs comerciais, sempre visando também o desenvolvimento de novas tecnologias que possam ser inseridas no gerenciamento dos motores.

Nos últimos projetos também já foram utilizadas tecnologias atuais do mercado automotivo como flex-fuel e sistemas de cruise control. Esses projetos mostram que estas unidades de gerenciamento são capazes de controlar um motor de combustão interna de forma eficaz, muito próximo das aplicações originais.

\section{ABREVIATURAS}

$\begin{array}{ll}\text { AN } & \text { Analógica } \\ \text { AUTOSAR } & \text { Automotive Open System Architecture } \\ \text { CAN } & \text { Control Area Network } \\ \text { CI } & \text { Circuito Integrado } \\ \text { DSP } & \text { Digital Signal Processor } \\ \text { ECU } & \text { Electronic Control Unit } \\ \text { FATEC } & \text { Faculdade de Tecnologia do Estado de São Paulo } \\ \text { GM } & \text { General Motors } \\ \text { JTAG } & \text { Joint Test Access Group } \\ \text { LCD } & \text { Liquid Crystal Display } \\ \text { LED } & \text { Light Emitting Diode } \\ \text { MAP } & \text { Manifold Air Pressure } \\ \text { MUX } & \text { Multiplexador }\end{array}$




$\begin{array}{ll}\text { OBD-II } & \text { On-Board Diagnostic II } \\ \text { PIC } & \text { Peripheral Interface Controller } \\ \text { PWM } & \text { Pulse Width Modulation } \\ \text { RPM } & \text { Rotações por Minuto } \\ \text { RTOS } & \text { Real Time Operating System } \\ \text { SPI } & \text { Serial Peripheral Interface } \\ \text { TPS } & \text { Throttle Position System } \\ \text { UART } & \text { Universal Asynchronous Receiver/Transmitter } \\ \text { uC } & \text { Microcontrolador } \\ \text { UCM } & \text { Unidade de Controle do Motor } \\ \text { USART } & \text { Universal Synchronous Asynchronous Receiver Transmitter } \\ \text { USB } & \text { Universal Serial Bus } \\ \text { USP } & \text { Universidade de São Paulo }\end{array}$

\section{REFERÊNCIAS}

[1] PEREIRA, BRUNO; Evolução de uma Unidade de Gerenciamento Eletrônico de um motor VW 2.0L e Desenvolvimento de Controle de Cruzeiro: Projeto Otto IV.

Universidade de São Paulo, 2017.

[2] ALVES, ELTON; JATO, FELIPE; HIROKI, GUSTAVO. Desenvolvimento de uma Unidade de Gerenciamento Eletrônico para Motor Volkswagen 1.6L. Fatec Santo André, 2016.

[3] DIAS, Bruno; Kitani, Edson; Silva, Cynthia; Guedes, Marcos; Laganá, Armando; Soares, André; Pereira, Bruno. Projeto do Hardware de uma Unidade Eletrônica de Controle (ECU) para Gerenciamento de Motores à Combustão Interna. Congresso SAE internacional, 2011-36-0320.

[4] Albaladejo, Felipe. Desenvolvimento de uma Unidade de Gerenciamento Eletrônico para Motores de Combustão Interna ciclo Otto. Universidade de São Paulo, 2013.

[5] PEREIRA, BRUNO; Unidade de Gerenciamento Eletrônico de um Motor Volkswagen 2.0L. Congresso SAE internacional, 2014-36-0442.

[6] MOSCARDINI, DEMERSON; MATA, VALTER; Unidade de Gerenciamento Eletrônico de motor ciclo Otto Microchip PIC Volkswagen 2.0L: Plataforma de desenvolvimento III. Fatec Santo André, 2014.

[7] DIAS, BRUNO; Plataforma didática de injeção eletrônica para controle de motores a combustão interna. FATEC Santo André, 2011.

[8] DIAS, BRUNO; Unidade microcontroladora para gerenciamento eletrônico de um motor de combustão interna ciclo Otto. Universidade de São Paulo, 2015. 